\title{
CloudZone: towards an integrity layer of cloud data storage based on multi agent system architecture
}

\begin{abstract}
The computing power in a cloud computing environments is supplied by a collection of data centers, or cloud data storages (CDSs) housed in many different locations and interconnected by high speed networks. CDS, like any other emerging technology, is experiencing growing pains. Data integrity checking of data and data structures has grown in importance recently in cloud computing due to the expansion of online cloud services, which have become reliable and scalable. In this paper we propose an integrity layered architecture of a typical cloud based on MAS architecture consists of two main layers cloud resources layer (cloud serverside) and MAS architecture layer (cloud client-side). At the cloud resources layer there exist massive physical cloud resources (storage servers and cloud application servers) that power the CDS. MAS's architecture layers consist of two agents: Cloud Service Provider Agent (CSPA) and Cloud Data Integrity Backup Agent (CDIBA). This layered architecture named as ñCloudZoneò. A prototype of our proposed ñCloudZoneò will be designed using Prometheus Methodology and implemented using the Java Agent Development Framework Security (JADE-S).
\end{abstract}

Keyword: Cloud computing; Cloud data storage; Cloud service provider; Integrity; Multi agent system 